\title{
Localization of Guanylate Cyclase Receptor, Inositol Trisphosphate Receptor, and Calmodulin in Boar Spermatozoa
}

\author{
Ayele Gugssa, Ava Williams, Rakeb Tilahun, William Eckberg \& Winston Anderson \\ Department of Biology, Howard University, Washington, DC 20059, USA \\ Tel: 202-806-6950Ｅ-mail:wanderson@howard.edu
}

\author{
Balwant Ahluwalia \\ Obstetrics and Gynecology, Howard University \\ Washington, DC 20059, USA \\ Tel: 202-865-1662 E-mail: bahluwalia@howard.edu \\ Lalita Kaul \\ Community Health and Family Medicine \\ Howard University, Washington, DC 20059, USA \\ Tel: 202-806-9840Ｅ-mail: 1kaul@howard.edu \\ Diana Broomfield \\ Department of Obstetrics \& Gynecology \\ Howard University, Washington, DC 20059, USA
}

\begin{abstract}
Several proteins present on the sperm membranes serve as signal transducers facilitating sperm maturation, capacitation, acrosome reaction and fertilization. Among them, guanylate cyclase (GC), the inositol trisphosphate receptor $\left(\mathrm{IP}_{3} \mathrm{R}\right)$ and calmodulin have been reported rather extensively. Although biochemical data clearly suggest their roles in the fertilization process, the precise location of these proteins in the sperm membranes have not been adequately investigated and need clarification. Using immunolocalization procedures we report that guanylate cyclase receptors (GCR) are localized on the apical region of the acrosome and on the postacrosomal plasmalemma, while $\mathrm{IP}_{3} \mathrm{Rs}$ are present in the neck region of the sperm and at lower density along the axonemal membrane. In contrast, calmodulin is restricted to the postacrosomal, basal nuclear membrane and the neck region that corresponds to the redundant nuclear envelope and ER vesicles, with some reaction along the middle piece and tail membranes. Colocalization of these proteins on the sperm membrane together with biochemical data as reported previously provide further insight into their role in the spermatozoan motility and possibly the fertilization process.
\end{abstract}

Keywords: Boar sperm, Calmodulin, Inositol trisphosphate receptor, Guanylate cyclase

\section{Introduction}

It is well established that a complex set of biochemical events in mammalian spermatozoa is triggered by several proteins, present on sperm membranes, that are involved in capacitation, acrosome reaction, and fertilization (deKretser et al., 1998). Receptors for cyclic guanosine monophosphate (cGMP) [Bentley and Garbers, 1986, Revelli et al., 2001, 2002, Willipinski-Stapelfeld 2004], cyclic adenosine monophosphate (cAMP) [Kurose et al., 1987, Chang et al., 1990, Harrison and Miller 2000], and inositol 1,4,5-trisphosphate (IP3), [Schilling et al., 1992, Walensky and Snyder, 1995] have been implicated in these processes. The actual mediator of signal transduction appear to be an increase in intracellular $\mathrm{Ca}^{2+}$ through voltage gated calcium channels into the sperm membranes (Bentley, et al., 1987, Arab et al., 1990, Verma et al., 1992). cGMP appears to be essential in transporting calcium through ion gated channels into the sperm membranes 
(Ramaroa and Garbers. 1985, Wedel and Garbers 2001, Revelli et al., 2002, Sharma 2002), and guanylate cyclase, the ubiquitous enzyme, catalyzes the conversion of GTP to cGMP mediated by nitric oxide (NO) [Bentley et al., 1986]. The peptide mediated protein phosphorylation in spermatozoan membranes appears to be due to enhanced formation of cGMP (Bentley et al., 1986). In capacitated spermatozoa it is known that the acrosome reaction (AR) can be induced by $\mathrm{Ca}^{2+}$ ionosphere A23187, an essential step in mammalian fertilization (Jaiswal et al 1998). IP3 has been implicated in the regulation of $\mathrm{Ca}^{2+}$ from intracellular membrane stores during hyperactivated motility in several sperm species (Suarez and Osman, 1987, Ho and Suarez, 2001, 2003). IP3 may also be implicated in the acrosome reactions in several species (Bird et al.; 1991). Increase in intracellular $\mathrm{Ca}^{2+}$ through IP3-gated channels may therefore be associated with a signaling cascade leading to cell motility, the acrosome reaction, and capacitation (Brass and Joseph, 1985).

The localization GCR, IP3R and calmodiulin on the sperm membranes in boar sperm will give us further insight into their role in the process leading to the various sperm functions.

\section{Methods and Materials}

\subsection{Preparation of Boar Sperm}

Boar semen was obtained commercially (Zierke Morris MN.). Only those samples with $90 \%$ or more sperm motility were used for the study. Sperm were isolated from semen using PBS-Percoll gradient solution. The gradient was prepared by layering $10 \mathrm{ml}$ of $35 \%$ Percoll over $10 \mathrm{ml}$ of $70 \%$ Percoll solution. Approximately $4.5 \times 10^{8}$ sperm in $10 \mathrm{ml}$ Tyrode's solution was layered over $35 \%$ Percoll solution, in a $50 \mathrm{ml}$ centrifuge tube and centrifuged at 2,000 x g for 5 min followed by $4,000 \mathrm{x}$ g for $10 \mathrm{~min}$. The fluffy mass of sperm near the middle of $70 \%$ Percoll layer was aspirated using a pipette and transferred to a clean centrifuge tube. The sperm were resuspended in PBS and recentrifuged at $400 \mathrm{xg}$ for $15 \mathrm{~min}$.

\subsection{Preparation of anti ROS-GC1 Antibody}

The anti-ROS-GC1 antibody was a gift from Dr. Teresa Duda. (Penn College of Optometry, Elkine Park, PA). The antibody was prepared in rabbit against c-terminal portion of the protein amino acid 1016-1054. $\mathrm{Ca}^{2+}$ ROS-GS1 antibody has been reported in the testes (Jankowska et al., 2007). It is known that Cyclic GMP produced by ROS-GC1 regulates the activity of nucleotide gated channel which is present in the testes (Sharma 2002).

\subsection{Immunocytochemical Procedures}

Sperm were fixed in 4\% paraformaldehyde in PBS for one hour, washed with PBS and blocked in PBS containing 1\% nonfat dry milk. The sperm were washed three times with PBS and incubated with the rabbit monoclonal antibodies at 1:500 dilution, GC (guanylate cyclase ROS-GC1), rabbit monoclonal antibodies against $\mathrm{IP}_{3} \mathrm{R} 1$ (Abcam Inc, Cambridge, MA), and mouse anti Calmodulin (Abcam Inc., Cambridge, MA) antibodies at 1:500 dilution for one hour. Sperm were washed three times with PBS and incubated with secondary goat and rabbit antibodies conjugated to Alexa fluorophores were obtained from Invitrogen (Molecular Probes). 4',6'-Diamidino-2-phenylindole (DAPI) [Ted Pella, Redding, CA] was used as counter staining for the nucleus, and images were obtained in a Zeiss $10 \mathrm{Z0}$ fluorescence microscope

\subsection{Transmission Electron Microscopy and Immunolabeling}

Washed sperm isolates were centrifuged at $4000 \mathrm{X} \mathrm{g}$ for 5 minutes, pelleted and fixed by immersion in $2 \%$ paraformaldehyde-glutaraldehyde fixative in $0.1 \mathrm{M}$ sodium cacodylate buffer $(\mathrm{pH}$ 7.2) for 1 hour. The specimen was postfixed in $2 \% \mathrm{OsO}_{4}$ for $1 \mathrm{hr}$, dehydrated in a graded series of ethanol to $100 \%$, and infiltrated using 1:1 alcohol and Spurrs (Ted Pella, Redding, CA) for 2 hrs and embedded in Spurrs. Ultrathin sections ( $\sim 90 \mathrm{~nm})$ were cut using a diamond knife and stained with $1 \%$ uranyl acetate and $1 \%$ lead citrate. For immunolabeling the sections on nickel grids were blocked in $1 \%(\mathrm{w} / \mathrm{v})$ bovine serum albumin for $30 \mathrm{~min}$ and washed three times with water before floated onto affinity-purified primary antibodies (1:500 in blocking buffer) for 1 hour. The grids were washed 5 times (10 min each wash) with water and incubated in secondary antibody $1: 500(15 \mathrm{~nm}$ Protein A-gold colloidal particles conjugated-anti-rabbit antibody (E-Y Labs, Inc., San Mateo, CA) for $45 \mathrm{~min}$ and washed 5 times with water. The grids were stained briefly with $1 \%$ uranyl acetate (aqueous) and lead citrate and then rinsed in three changes in water and examined by electron microscopy.

\section{Results}

\subsection{Localization of Guanylate Cyclase Receptors}

To determine the localization of the components of the ROS-GC1 in boar sperm, immunofluorescence analysis was carried out with specific antibodies against ROS-GC1. Staining for ROS-GC1 was consistently observed in the neck region of the sperm, with weaker staining along the middle piece and at the apical surface of the acrosome (Fig. 1b). Control specimens revealed no labeling (Fig. 1a).

This localization was confirmed by electron microscopy. A high density of Protein A-gold particles indicating the presence of GCR was observed on the plasmalemma over the middle piece of the sperm (Fig. 2A). Control specimens showed no labeling by the immunogold procedure (Fig. 2B). 


\subsection{Localization of Inositol Trisphosphate Receptors}

Immunofluorescence analyses were carried out with specific antibodies against $\mathrm{IP}_{3} \mathrm{R}$. $\mathrm{IP}_{3} \mathrm{Rs}$ were observed predominantly in the postnuclear neck region, with light staining of the middle piece region (Fig. 3). Control specimens showed no reaction. Regions stained for $\mathrm{IP}_{3} \mathrm{R}$ corresponded to clusters of vesicles and nuclear membrane folds also observed in this region (Fig. 3 C, D).

\subsection{Calmodulin}

Fluorescence staining consistently revealed calmodulin in the neck region and surrounding the basal surface of the nucleus (Fig. 4A, C). Control specimens showed no fluorescence (Fig. 4B)

\section{Discussion}

The major finding of the study is that there is a colocalization of signal transduction proteins on the sperm membranes. Close association of $\mathrm{IP}_{3} \mathrm{R}$ and GCR in sperm membrane supports the suggestion made by Suarez and coworkers that IP3 mediated release of $\mathrm{Ca}^{2+}$ from calmodulin stores is related to the control of gated calcium channels during sperm motility and hyperactivated motility (Ho and Suarez, 2001, 2003). These processes may also be operational during fusion of sperm with oocyte membranes at the time of fertilization (Liu and Barker 1997, Revelli et al., 2002). The role of GC is well defined and several studies confirm that $\mathrm{GC}$ regulates not only the gated $\mathrm{Ca}^{2+}$ channel but it also plays an important role as a chemotactic agent during sperm oocyte fusion in sea urchin (Santos-Sacchi and Gordon 1980, Bentley et al., 1986, Cohen-Dayag et al., 1995). It is suggested that initiation of the $\mathrm{IP}_{3} / \mathrm{cGMP}$ signal transduction events control the release of $\mathrm{Ca}^{2+}$ from calmodulin bound calcium which results in activation of protein kinase $\mathrm{C}(\mathrm{pKC})$ causing phosphorylation of enzymes involved in spermatozoan movement.

Depending upon the species of sperm and reaction conditions, signal transduction proteins and receptors appear to have different structural localizations. For instance the IP3 signaling system is localized on the anterior acrosomal region of the mouse, hamster, dog and bull sperm and the acrosomal cap of rat sperm where it might be related to IP3-gated $\mathrm{Ca}^{2+}$ channels (Walensky and Snyder, 1995; Ho and Suarez, 2001). A focus of $I_{3} \mathrm{R}$ is localized at the proximal middle piece of the rat sperm, suggesting that an $\mathrm{IP}_{3}$-gated calcium store at the proximal middle piece may function in regulation of sperm motility. In contrast, $\mathrm{IP}_{3} \mathrm{R}$ was not observed in the proximal middle piece of the mouse, hamster or dog sperm (Walensky and Snyder, 1995). A recent study by Suarez and co-workers (Ho and Suarez, 2003) found $\mathrm{IP}_{3} \mathrm{R}$ to be present primarily in the neck region of the bull sperm that corresponds to a plethora of vesicles, mostly associated with the nuclear envelope. Localization of $\mathrm{IP}_{3} \mathrm{R}$ at these sites relates to $\mathrm{Ca}^{2+}$ storage in the neck region of sperm. Our results using boar sperm clearly corroborates Suarez's findings, but shows vesicular clusters that might be related to the endoplasmic reticulum (ER).

A phosphotidyl inositol signaling pathway has been observed in sperm membranes where hydrolysis of phosphatidylinositol 4,5-bisphosphate $\left(\mathrm{PIP}_{2}\right)$ generates $\mathrm{IP}_{3}$ and diacyl glycerol (DAG), leading to the activation of $\mathrm{pKC}$. Indeed, $\mathrm{IP}_{3}$ activated $\mathrm{Ca}^{2+}$ release from nuclear membrane and ER vesicles may be related to activation of plasma membrane localized $\mathrm{pKC}$, and subsequent phosphorylation of intracellular proteins necessary for sperm function.

Numerous studies have demonstrated that extracellular $\mathrm{Ca}^{2+}$ has a regulatory role in the control of motility in sperm of several species (Breitbart, 2002). Sperm membrane-associated $\mathrm{Ca}^{2+}$ stores, a $\mathrm{Ca}^{2+}$ ATPase or ATP $\mathrm{Ca}^{2+}$ pump may be involved in maintaining motility of spermatozoa. Immunocytochemical studies by Suarez and coworkers (Ho and Suarez, 2003) show strong presence of calcium-storage proteins (calmodulin, calreticulin) in the neck region of hyperactivated bull sperm. Our results confirm previous findings showing intense labeling for calmodulin in the neck and in regions surrounding the basal region of the boar sperm nucleus. These results also suggest the interrelatedness of IP3Rs at postnuclear vesicular and ER membranous sites and calmodulin storage sites. Other studies by Camatini and coworkers (1986) demonstrate calmodulin in the acrosomal content and in the equatorial and postacrosomal regions of boar sperm.

Our localization study and other studies described earlier suggest that membrane borne GC, primarily in the neck region of the boar sperm, modulates the influx of extracellular $\mathrm{Ca}^{2+}$, the synthesis of $\mathrm{IP}_{3}$ that result in release of $\mathrm{Ca}^{2+}$ from postnuclear membranous and ER sites and possible activation of $\mathrm{pKC}, \mathrm{Ca}^{2+}$ pumps and $\mathrm{Ca}^{2+}$-activated ATPases to control axonemal and mitochondrial reactions leading to spermatozoan motility. The question that still remains to be answered is whether colocalization of proteins identified in the sperm membranes act independent of each other or synchronize their functions in unison to regulate spermatozoan motility, capacitation, acrosome reaction and fusion with the oocyte. This idea is speculative and needs further investigation.

\section{References}

Arab, N., Shibata, S.H., \& Ghishan, F.K. (1990). Ontogeny of mitochondrial calcium transport in spontaneously hypertensive (SHR) and WKY rats. Developmental Physiology, 14, 59-67.

Bentley, J.K., \& Beavo, J.A. (1992). Regulation and function of cyclic nucleotide. Current Opinion in Cell Biology 4, 233-40. 
Bentley, J.K., \& Garbers, D.L. (1986). Receptor-mediated responses of plasma membranes isolated from Lytechinus pictus spermatozoa. Biology of Reproduction, 35: 1249-1259.

Bentley, J.K., Tubb, D.J., \& Garbers, D.L. (1986). Receptor-mediated activation of spermatozoan guanylate cyclase. Journal of Biological Chemistry, 261, 14859-14862.

Bird, G.S.J., Rossier, M.F., Hughes, A.R., Shears, S.B., Armstrong D.L. \& Putney, J.W. (1991). Activation of C ${ }^{2+}$ entry into acinar cells by a nonphosphorylatable inositol trisphosphate Nature, 352, 162-165.

Brass, L.F. \& Joseph, S.K. (1985). A role for inositol trisphosphate in intracellular $\mathrm{Ca}^{2+}$ mobilization and granule secretion in platelets. Journal of Biological Chemistry, 260, 15172-15179.

Breitbart, H. (2002). Intracellular calcium regulation in sperm capacitation and acrosomal reaction. Mol. Cell.

Endocrinol., 187: 139-144

Camatini, M., Anelli, G., \& Casale, A. (1986). Immunocytochemical localization of calmodulin in intact and acrosome-reacted boar sperm. Eur. J. Cell Biol., 41: 89-96.

Chang, C.H., Kohse, K.P., Chang, B., Hirata, M., Jiang, B., Douglas, J.E., \& Murad, F. (1990). Characterization of ATP stimulated guanylate cyclase activation in rat lung membranes. Biochimica et Biophysica Acta, 1052, $159-165$.

Cohen-Dayag A., Tur-Kaspa I., Dor J., Mashiach S., \& Eisenbach M. (1995). Sperm capacitation in humans is transient and correlates with chemotactic responsiveness to follicular factors. Proceedings of the Natlional Academy of Sciences, USA. 92, 11039-11043.

deKretser, D.M., Loveland, K.L., Meinhardt, A., Simorangkir, D., \& Wreford (1998). Spermatogenesis. Human Reproduction, 13: 1-8

Harrison, N.A., \& Miller, N.G. (2000). cAMP dependent protein kinase control of plasma membrane lipid architecture in boar sperm. Molecular Reproduction and Development, 55, 220-228.

Ho, H.C., \& Suarez, S.S. (2001) An inositol 1,4,5-trisphosphate receptor-gated intracellular $\mathrm{Ca}^{2+}$ store is involved in regulating sperm hyperactivted motility. Biology of Reproduction, 65, 1606-1615.

Ho H.C., \& Suarez, S.S. (2003). Characterization of the intracellular calcium store at the base of the sperm flagellum that regulates hyperactivated motility. Biology of Reproduction, 68, 1590-1596.

Jaiswal, B.S., Cohen-Dayag, A., Tur-Kaspa, I., \& Eisenbach, M. (1998). Sperm capacitation is, after all, a prerequisite for both partial and complete acrosome reaction. FEBS Letters, 427, 309-313.

Jankowska, A., Burczynska, B., Duda, T., Warchol, JB., \& Sharma, RK. (2007). Calcium-modulated rod outer segment membrane guanylate cyclase type 1 transduction machinery in the testes. Journal of Andrology, 28, 50-58.

Kurose H., Inagami T., \& Ui, M. (1987). Participation of adenosine 5'-triphosphate in the activation of membrane-bound guanylate cyclase by the atrial natriuretic factor. FEBS Letters, 219, 375-379.

Liu, DY., \& Barker, HWG. (1997). Protein kinase C plays an important role in the human zona pellucida-induced acrosome reaction. Molecular Human Reproduction, 3, 1037-1043.

Ramaroa, CS., \& Garbers, DL. (1985) Receptor-mediated regulation of guanylate cyclase activity in spermatozoa. Journal of Biological Chemistry, 260, 8390-8396.

Revelli, A., Costamagna, C., Moffa, F., Aldieri, E., Ochetti, S., Bosia, A., Messpbrio, M., Lindblom, B., \& Ghigo, D. (2001). Signalling pathway of nitric oxide induced reaction in human sperm. Biology of Reproduction, 64, $1708-1712$.

Revelli, A., Ghigo, D., Moffa, F., Massobrio, M., \& Tur-kaspa, I, (2002). Guanylate cyclase sctivity and sperm function. Endocrine Review, 23, 484-494.

Schilling, W.P., Cabello, O.A., \& Rajan, L. (1992). Depletion of the inositol 1.4,5 trisphosphate-sensitive Ca ${ }^{2+}$ store in vascular endothelial cells activates the agonist sensitive $\mathrm{Ca}^{2+}$ influx pathway. Biochimica et Biophysica Acta, 284, 521-530.

Santos-Sacchi, J., \& Gordon, M. (1980). Induction of the acrosome reaction in guinea pig spermatozoa by cGMP analogues. Journal of Cell Biology, 65, 798-803.

Sharma R.K. (2002). Evolution of the membrane guanylate cyclase transduction system. Molecular and Cellular Biochemistry, 230, 3-30.

Suarez S.S. \& Osman, R.A. (1987). Iniatiation of hyperactivated flagellar bending in mouse sperm within the female reproductive tract. Biology of Reproduction, 36, 1191-1198.

Wedel, B.J. \& Garbers, D.L. (2001). The guanylate cyclase family at Y2K Annual Review of Physiology, 63, $215-33$. 
Verma, A., Hirsch, D.J., \& Snyder, H. (1992). Calcium pools mobilized by calcium or inositol 1,4,5-triphosphate are differentially localized in rat heart and brain Molecular Biology of the Cell, 3, 621-631.

Walensky, L.D. \& Snyder, S.H. (1995). Inositol 1,4,5 triphosphate receptors selectively localized to the acrosome of mammalian sperm. Journal of Cell Biology, 130, 857-869.

Willipinski-Stapelfeld, B., Lubberstedt, J., Stelter, S., Vogt, K., Mukhopadhyay, A.K., \& Muller, D. (2004). Comparative analysis between cyclic AMP signaling in human sperm. Human Reproduction, 2004 10, 543-552.
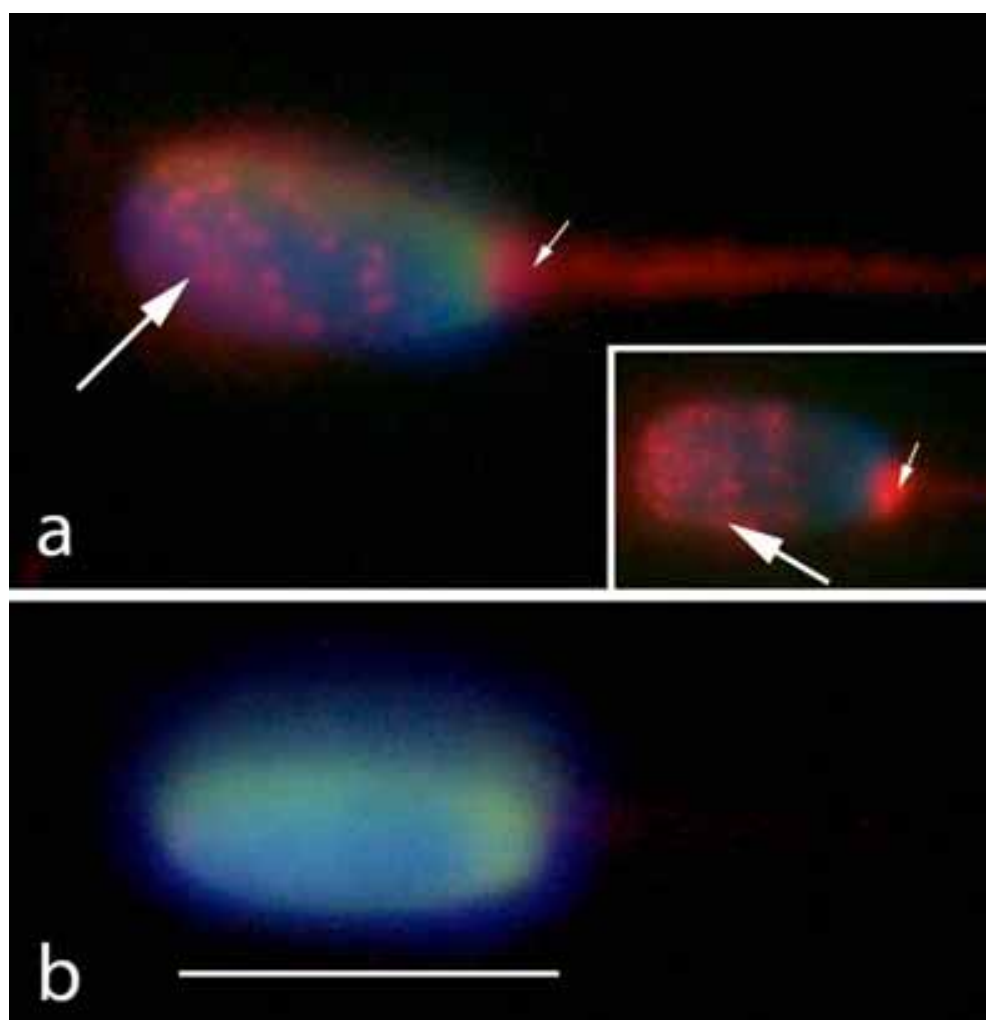

Figure 1. Localization of GCR in mature boar sperm. (a) Sperm stained with DAPI (blue) and antibody to GCR (red) showing the enzyme in the postnuclear neck region, (smaller arrows) and on the plasmalemma at the apical surface of the acrosome, (larger arrows). (b) DAPI-stained control sperm show no reaction. Scale bar $=10 \mu \mathrm{m}$.

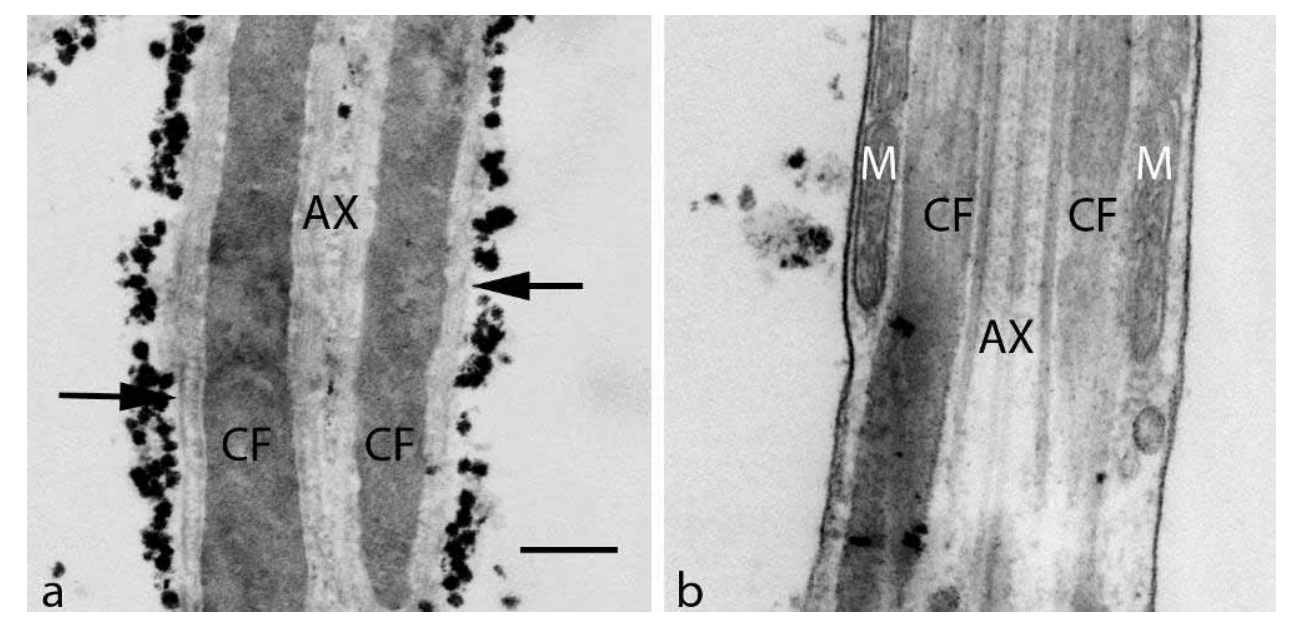

Figure 2. The ultrastructure of the middle piece and tail region of boar sperm are illustrated (a) Electron microscope micrographs showing localization of GCR by immunogold labeling on the membrane around the middle piece (arrows). $\mathrm{AX}=$ flagellar axoneme; $\mathrm{M}=$ mitochondrion; $\mathrm{CF}=$ coarse fibers. Control specimen (b) shows no labeling. Scale bar = $0.5 \mu \mathrm{m}$. 

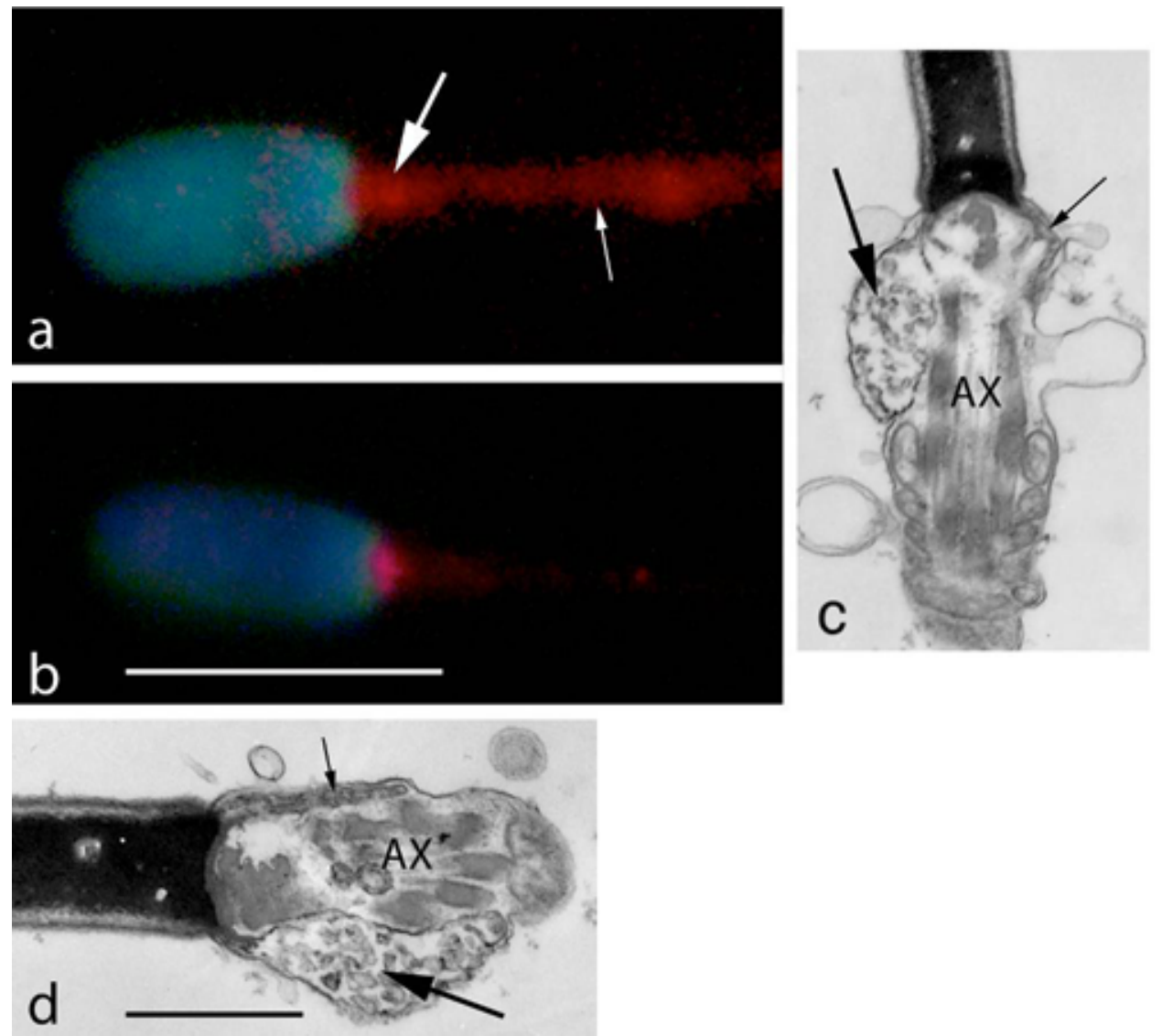

Figure 3. Localization of $I P_{3} R$ in mature sperm. Boar sperm were probed with antibody to $I_{3} R$. (a,b) Sperm show $\operatorname{IP}_{3} R$ staining in the neck region (larger arrows), with some staining in the middle piece (smaller arrow). Scale bar $=10 \mu \mathrm{m}$. (c,d) Portions of the redundant nuclear envelope (smaller arrows) and membrane-bound vesicles (larger arrows) abound in the region. $\mathrm{AX}=$ flagellar axoneme. Scale bar $=1 \mu \mathrm{m}$.
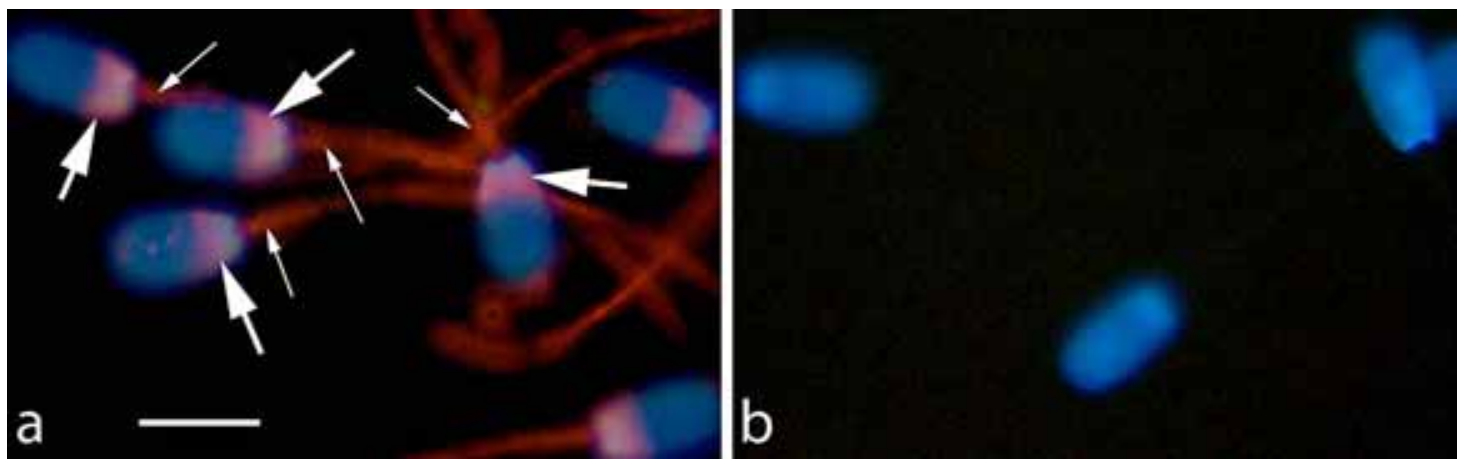

Figure 4. Localization of calmodulin in mature sperm. Fluorescence staining consistently revealed that calmodulin was localized in the neck (larger arrows) and postacrosomal (smaller arrows) regions (a). Control specimens (b) showed no labeling. Scale bar $=10 \mu \mathrm{m}$. 